\title{
Application of Precede-Proceed Model Based Education for Prevention of Osteoporosis Risks among Perimenopausal Women
}

\section{Amany Ahmed Mohamed ${ }^{1}$, Mohamed Abd-Elsalm Mohamed ${ }^{2}$, Soad Abd-Elsalam $\operatorname{Ramadan}^{3}$ and Hemmat Mostafa Elbana ${ }^{4}$}

(1) Assistant lecturer of Obstetrics and Gynecological Nursing, Faculty of Nursing, Benha University, Egypt, (2) Professor of Obstetrics and Gynecological Medicine - Faculty of Medicine Benha University, Egypt, (3) Professor of Obstetrics and Gynecological Nursing, Faculty of Nursing -Benha University, Egypt and (3) Assistant Professor of Obstetrics and Gynecological Nursing, Faculty of Nursing -Benha University, Egypt

\begin{abstract}
Background: Osteoporosis is a major public health problem worldwide especially among menopausal women in the world, which can be prevented or its onset can be delayed through health education and lifestyle changes. Aim of study: Was to evaluate the effect of application of PRECEDE-PROCEED model (PPM) based education for prevention of osteoporosis risks among perimenopausal women. Design: A quasi-experimental design was used to achieve the aim of the study. Setting: This study was conducted at the Administrative building of Benha University hospital (administrator employee). Sample: A purposive sample was carried out in the study according inclusion criteria. The sample consists of 109 perimenopausal women were divided randomly into two groups (control group compromising 54 women and study group compromising 55 women). Tools: Two main tools were used; Self-administered questionnaire and Construction of Precede-Proceed model. Results: There was a highly statistically significant difference was observed in all construction of Precede-Proceed model of the study group compared with the control group after application of PRECEDE - PROCEED model $(\mathrm{p}<.001)$. Conclusion: Application of PRECEDE-PROCEED model was effective in prevention of perimenopausal women's predisposing factors of osteoporosis, healthy practices and activities, reinforcing factors, enabling factors which supported the present study hypothesis. Recommendations: It is crucial to conduct an extensive future studies with larger sample probability sizes of perimenopausal women to enhance the accuracy of the findings, Also application of PRECEDE-PROCEED Model Based Education program among perimenopausal women in health system to increase the level of awareness towards osteoporosis and its prevention.
\end{abstract}

Key word: Osteoporosis, Perimenopause, PRECEDE - PROCEED model, Prevention

\section{Introduction}

Perimenopause transition begins several years before menopause. It's the time when the ovaries gradually begin to make less estrogen. It usually starts in women's $40 \mathrm{~s}$, but can start in $30 \mathrm{~s}$ or even earlier. Perimenopause lasts up until menopause, the point when the ovaries stop releasing eggs. In the last 1 to 2 years of perimenopause, this drop in estrogen speeds up. At this stage, many women have menopause symptoms. The average length of perimenopause is 4 years, but for some women this stage may last only a few months or continue for 10 years. 
Perimenopause ends when women have gone 12 months without having period (Huang et al., 2020).

Throughout the Perimenopausal transition, some changes in body may take place as: Irregular periods. As ovulation becomes more unpredictable, the length of time between periods may be longer or shorter, flow may be light to heavy, and may skip some periods, hot flashes and sleep problem and mood changes (Falkingham et al., 2020). Loss of bone with declining estrogen levels, start to lose bone more quickly than replacing, increasing risk of osteoporosis, is a disease that causes fragile bones (Jalil et al., 2021).

Osteoporosis is an irreversible, systemic skeletal disorder that affects bone density and quality, leading to bone fragility and increased risk of fractures. One in two women and one in five men over the age of 50 are expected to have a bone fracture, as a result of poor health of bones. Owing to the fact that people are usually not aware that bones are fragile until a time of a first fracture and thus it is known as a 'silent disease'. The fractures could result in disability, poor quality of life, and premature mortality, in addition to economic consequences (Jakimiuk\&Jackson, 2020).

Nurses can play an important role in the care of women with osteoporosis by educating women about the good dietary habits, well balanced diet that high in calcium and vitamin $\mathrm{D}$, as well as life style change factors that contribute to bone loss, such as cigarette smoking, excessive caffeine intake, and the importance of following the recommended medical regimen. Nurses are also concerned about how to prevent falls and thus reduce the risk of fractures. Suggestion to increase safety in the home include adequate lightening and voiding objects that might increase falls, such as loose electrical cords or rugs with nonskid backing (Mckinney,2020).

\section{Significance of the study}

In this study, precede-proceed model was used to implement health education and health promotion interventions for the prevention of osteoporosis risks. This model as an evaluation framework for health education and health promotion planning, individual or community interventions (Recker, 2019).

In the Egypt, menopausal women constitute a considerable sector of the whole population .percentage of women at menopausal age to all women to the whole population is $20.5 \%$. Recent statistics in Egypt proved that about 4.7 millions of women at age of 45 years or more have osteoporosis .This number is increased to 7.7 million by year of 2020 and to 13 million by the year 2030 (Abd El Rahman et al.,2019).

\section{Aim of the study}

This study aimed to evaluate the effect of application of PRECEDEPROCEED model based education for prevention of osteoporosis risks among perimenopausal women.

\section{Research hypothesis:}

Application of precede-proceed model based education would help to prevent osteoporosis risks among perimenopausal women.

\section{Subjects and method}

Study design:

A quasi-experimental design was utilized to fulfill the aim of this study.

\section{Study setting:}

The study was conducted at Administrative building of Benha university 
hospital in Benha city (administrator perimenopausal employee).

\section{Sample:}

Sample type: A purposive sample

Inclusion criteria: Age 42-55, natural perimenopausal women, agreed to participate in the study, had telephone number.

Exclusion criteria: Premature menopause, diagnosis of osteoporosis, predisposing factor as menopause (History of hysterectomy and oophorectomy).

Sample size: Total number of perimenopausal women at Benha University hospital was 363 women $30 \%$ of total number (109 perimenopausal women) were being divided randomly in to two groups (study group was comprised of 55 women and control group was comprised of 54 women).

\section{Sample technique:}

A Perimenopausal woman was chosen by simple random sample. The researcher was visited the study settings and, explained the purpose of the study to perimenopausal women and the visit was repeated 3times/weekly until predetermined number completed.

\section{Tools for data collection:}

Two main tools were used for data collection:-

Tool I: Self-administered questionnaire: It was designed by the researcher after reviewing related literature (Azar et al., 2018) and under guidance of supervisors; it was written in an Arabic language in the form of close and open- ended questions. and consisted of two parts:

First part: included:

Section one: Socio-demographic characteristics such as (age, residence, educational level, marital status, nature of work, monthly income).
Section two: anthropometric data: The researcher weighed each woman and measured her height to estimate $(\mathrm{BMI})=$ weight (kg) / height (m) 2. Weight was measured in kilograms using a weight scale.

\section{BMI Categories:}

- Underweight $=<18.5$

- $\quad$ Normal weight $=18.5-24.9$

- $\quad$ Overweight $=25-29.9$

- $\quad$ Obesity $=$ BMI of 30 or greater

Section three: Obstetrical and gynecological history such as (gravida, parity, using of family planning methods and breastfeeding children).

Section four: Menstrual history such as (age of menarche, regularity of menstrual cycle, menstruation cessation and taking replacement hormones after menopause).

Second part: Healthy practices and activities questionnaires schedule: It was adapted from (Kuar, 2019). This questionnaires comprised of 33 items to measure healthy practices and activities of women physical activity in past month and was divided into seven dimensions; dietary habits (16 items), practicing exercise ( 3 items), exposure to the sun (1 item), smoking (1 item), Periodic check-up (2 items), taking medication (5 items) and reducing the risk of falling (5 items).

\section{Scoring:}

Each item had -point Likert's scale ranging from (0) to (2), each item scored as following: (2) if always done, (1) if sometimes done and (0) if never done. The total healthy practices and activities' score was classified into two levels as the following:

- Satisfactory level: $\geq 75 \%$

- Unsatisfactory level: $<75 \%$

Tool II: Precede-Proceed model constructs:

A-Predisposing factor included two parts 
Part One: knowledge regarding osteoporosis and its prevention. It consisted of 7 section of knowledge.

\section{Scoring system:}

Each item was assigned a score of (2) given when the answer was completely correct, a score (1) was given when the answer was incompletely correct and a score (0) was given when the answer was don't know or wrong. The total score of each part was calculated by summation of the scores of its items. The total score for the knowledge of a woman was calculated by the addition of the total score of all parts. As well as women' total knowledge score was classified as the following:

-Poor $\quad(<50 \%$ correct answers $)$

-Average (50\% to $<75 \%$ correct answers)

-Good ( $\geq 75 \%$ to $100 \%$ correct answers).

Part Two: Women's attitude regarding prevention of osteoporosis. (Modified likert scale) .This scale was adapted from (Ghaderi et al., 2010). It was translated into Arabic language to suit women's level of understanding and modified by the researcher to have broader study about perimenopausal women's attitude regarding prevention of osteoporosis. The questionnaire consisted of 12 items.

\section{Scoring system:}

To obtain the outcome of attitude scale, the items were judged according to three point likert scale continuum from disagree (0), to some extent (1), and agree (2). Women' total attitude score was expressed as a percentage and classified as the following:

- Negative attitude: $<75 \%$

- Positive attitude: $\geq 75 \%$

B: Enabling factor: was adapted from (Meselhy et al.,2016)The questionnaires were translated into Arabic language to suit women's level of understanding and consisted of 8 items.

\section{Scoring system:}

The answer choices to these statements were "always", "to some extent" and "never". The "always" answer was allocated a score of (2), "to some extent" was allocated a score of (1) and "never" was allocated a score of (0). The total score of enabling factors was classified into two levels:

-Strong factors: $\geq 60 \%$

-Weak factors: $<60 \%$

C: Reinforcing factor: was adapted from (Orabi, 2017), the questionnaire was translated into Arabic language to suit women's level of understanding and consisted of 7 items.

\section{Scoring system:}

The answer choices to these statements were "always", "to some extent" and "never". The "always" answer was allocated a score of (2), "to some extent" was allocated a score of (1) and "never" was allocated a score of (0). The total score of reinforcing factors was classified into two levels:

- $\quad$ Strong factors: $\geq 60 \%$

- Weak factors: < $60 \%$

\section{Tools validity:}

The validity of questionnaire was reviewed by jury experts :( 3 ) experts in the field of Obstetrics \& Gynecological Nursing to ascertain clarity, relevance, comprehensiveness and applicability of tools.

\section{Tool reliability:}

The reliability was done by Cronbach's Alpha coefficient test which revealed that: the internal consistency of knowledge assessment questionnaire was 0.81 , the internal consistency of attitude assessment scale was 0.79 and the internal consistency of enabling and reinforcing factor was 0.76 . 


\section{Pilot study}

A pilot study was conducted on $(10 \%)$ from the total sample (11 women) to test the clarity and applicability of the study tools as well as estimation of the time needed to fill the questionnaire. The modifications were done. Women involved in the pilot were excluded from the study and researcher adds another 11 perimenopausal women to prevent sample contamination.

\section{Ethical consideration:}

An official permission from the selected study setting was obtained for the fulfillment of the study. Approval of the faculty ethics committee for scientific research was obtained for the fulfillment of study. Each woman was informed about the purpose and benefits of the study at the beginning of interview and time throughout the study. An informal consent was obtained from each woman before starting data collection. Confidentiality was ensured throughout the study process, where personal data were not disclosed, and the women were assured that all data was used only for research purpose. Each woman was informed that, participation is voluntary and her withdrawal will not affect her care. The study didn't have any physical, social or psychological risk on the participants.

\section{Field work:}

The study was conducted through PPM phases. It was carried out from the beginning of June2019, and completed at the end of July2020 covering14 months. This study was conducted under the approval of the Faculty of Nursing Ethical Committee, Benha University. A written official approval to conduct this study was obtained from the Dean of faculty nursing to director of Benha university hospital. Other written official letter was taken and delivered to the director of Benha university hospital, in order to obtain their agreement to conduct the study after explaining its purpose. An informal consent was also taken from each woman for participation. The researcher conducted the study 3times/weekly (Saturdays, Mondays and Thursday); from 9.00 a.m. to 2.00 p.m. To fulfill the aim of this study, the following phases were followed; preparatory phase, assessment phase, planning phase, PRECEDE-PROCEED model implementation phase and evaluation phase.

\section{Preparatory phase:}

The preparatory phase was the first phase of the study, the researcher carried out through review of local and international related literature about the various aspects of the study problem. This helped the researcher to be acquainted with magnitude and seriousness of the problem, and guided the researcher to prepare the required data collection tools. The tool was distributed to experts in the field; the aim was to test its appropriateness, comprehensiveness, clarity, importance and applicability.

\section{Assessment phase:}

This phase encompassed meeting both control group and study group to collect questionnaire distribute and collect sociodemographic characteristics, baseline data about women's health practices and activities, knowledge and attitude regarding prevention of osteoporosis as well as enabling and reinforcing factors.

\section{Planning phase:}

Based on results obtained during assessment phase, the intervention program based on PRECEDE-PROCEED model was developed by the researcher after reviewing related literature in a form of printed booklet. The booklet was designed specifically for women, in simple Arabic language to suit their level of understanding and to satisfy the studied pregnant women's deficit knowledge 
and attitude regarding prevention of osteoporosis. It was illustrated by colored pictures. Sessions number and its contents, different methods of teaching, and instructional media were determined. Objectives were constructed to be attained after completion the intervention program.

\section{Implementation phase:}

PRECEDE-PROCEED

model

intervention program was conducted to study group through five sessions over a period of (20) weeks .Participants (study group) were classified to (18) sub group. Total time for all sessions to each woman took about three hours and half, each session took about 45-60 minutes. The sessions were repeated to each subgroup (3-4women/session).At the beginning of the first session women were oriented with the program contents. Each subgroup was informed about the time of the next sessions at the end of session. The subsequent session started by a feedback about the previous session and the objectives of the new session, using simple Arabic language to suit women' level of understanding. At the end of each session, women' questions were discussed to correct any misunderstanding.

The first session: included an orientation to the program, introduction about perimenopause, causes, risk factors, perimenopause symptoms, complication, methods of perimenopause management .The second session :included general knowledge about osteoporosis, causes, risks, prevention .The third session :included general knowledge about healthy behavior (periodic examination and investigations), importance of exercise, rate and types of exercises. The fourth session: included nutrition, and interpersonal relations. Women were taught about balanced diet, modifying unhealthy nutritional behaviors, strict intake of processed and sugar rich foods and carbohydrates, increasing intake of fresh vegetables and fruits, increasing dairy intake. As well as, maintaining weight control and reducing obesity. Social support and maintaining communications with others, overcoming the embarrassment and strengthening talking with the husband about perimenopause. The fifth session: included PPM, its phases and predisposing factors, reinforcing factors and enabling factors for prevention osteoporosis risks during perimenopause. The researcher gave women the educational booklet at the end of sessions.

Different methods of teaching were used such as discussion, demonstration and redemonstration. Instructional media included video contain all content of program and educational booklet about osteoporosis which constructed by the researcher in a simple Arabic language after reviewing the related literatures and based on women' deficit knowledge healthy practices and attitude regarding prevention of osteoporosis.

E-Break phase:

- The researcher contacted women in the study group by phone to ensure that women followed the guidelines and applied provided care effectively .The researcher provide any advice or care to women during break phase.

- Controlled group women followed by phone to avoid their drop out from the study, but no care provided to women during break phase to avoid study bias.

\section{Evaluation phase:}

The effectiveness of the PRECEDEPROCEED model intervention program was evaluated 3 months after implementation, using the same format of tools which used during the assessment phase (pre-posttest 
format) which were (tool I- Part 2 to measure healthy practices and activities of women physical activity and tool II to evaluate women's knowledge and attitude regarding prevention of osteoporosis and to evaluate the enabling and reinforcing factor).

Evaluation started first with control group then with study group to avoid bias. At almost time the researcher followed the women via telephone and what Sapp group due to Covid-19 circumstances. Women in control group were given the booklet.

\section{Statistical analysis:}

Data was verified prior to computerized entry. The Statistical Package for Social Sciences (SPSS version 22.0) was used for that purpose, followed by data tabulation and analysis. Descriptive statistics were applied (e.g., mean, standard deviation, frequency and percentages). Test of significance (t test, chi-square), Pearson correlation coefficients were used. A significant level value was considered when $\mathrm{p} \leq 0.05$. And A highly significant level value was considered when $\mathrm{p}<0.01$.

\section{Results}

Table (1): Clarifies that there was no statistically significant difference between control and study groups regarding sociodemographic characteristics (Age, residence, education, marital status, nature of work, monthly income $(p>0.05)$ reflected group homogeneity.

Table (2) Represents the anthropometric measurements of both study and control group, it showed that, the mean weight of both study and control groups were $80.95 \pm 11.24 \mathrm{~kg}$ and $77.54 \pm 12.24 \mathrm{~kg}$ respectively, the mean height of both study and control groups were $160.33 \pm 4.63 \mathrm{~cm}$ and
$161.70 \pm 3.76 \mathrm{~cm}$ respectively. In addition, the mean body mass index in both study and control groups were $31.12 \pm 4.30 \mathrm{~kg} / \mathrm{m} 2$ and $29.71 \pm 4.93 \mathrm{~kg} / \mathrm{m} 2$ respectively, this mean no significance difference in anthropometric data of both group ( $p>0.05)$ reflect homogenous.

Table (3): Illustrates that there was no statistically significant difference between control and study groups regarding all items of knowledge about osteoporosis before intervention $(\mathrm{P}>0.05)$. However, there was a highly statistical significant improvement were observed in the study group compared with the control group after intervention $(\mathrm{p}<$ 0.001).

Figure (1): Shows that $35.2 \%, 34.5$ of the studied women (control and study groups) had positive attitude regarding prevention of osteoporosis at pre intervention phases respectively that improved to $33.3 \%, 70.9 \%$ post intervention phases respectively.

Table (4): Represents that there was no statistically significant difference between control and study groups regarding all items of healthy practices and activities regarding dietary habits before intervention $(\mathbf{P}>\mathbf{0 . 0 5})$. However, there was a highly statistical significant improvement was observed in the study group compared with the control group after intervention $(\mathrm{p}<0.001)$.

Table (5): Clarifies that there was a highly statistically significant positive correlation between total knowledge score and total attitude of both study and control groups before and after intervention $(\mathrm{P}<$ 0.001). Moreover, there was a highly statistically significant positive correlation between total knowledge score and total healthy practices and activities of both study and control groups before and after intervention $(\mathrm{P}<0.001)$. 
Application of Precede-Proceed Model Based Education for Prevention of Osteoporosis Risks among Perimenopausal Women

Table (1): Distribution of the studied women (control and study groups) according to their socio-demographic characteristics $(\mathbf{n}=109)$.

\begin{tabular}{|c|c|c|c|c|c|}
\hline \multirow[t]{2}{*}{ Socio-demographic characteristics } & \multicolumn{2}{|c|}{$\begin{array}{c}\text { Control group } \\
n=54\end{array}$} & \multicolumn{2}{|c|}{$\begin{array}{l}\text { Study group } \\
\quad \mathrm{n}=55\end{array}$} & \multirow{2}{*}{$\begin{array}{c}\mathrm{X} 2 \\
\mathrm{p}- \\
\text { value }\end{array}$} \\
\hline & No & $\%$ & No & $\%$ & \\
\hline \multicolumn{6}{|l|}{ Age ( years) } \\
\hline $42-45$ & 13 & 24.1 & 18 & 32.7 & \multirow{4}{*}{$\begin{array}{l}1.80 \\
>0.05\end{array}$} \\
\hline $46-50$ & 23 & 42.6 & 17 & 30.9 & \\
\hline$\geq 51$ & 18 & 33.3 & 20 & 36.4 & \\
\hline Mean \pm SD & \multicolumn{2}{|c|}{$47.67 \pm 3.54$} & \multicolumn{2}{|c|}{$48.41 \pm 2.34$} & \\
\hline \multicolumn{6}{|l|}{ Residence } \\
\hline \multirow{2}{*}{$\begin{array}{l}\text { Rural } \\
\text { Urban }\end{array}$} & 34 & 63.0 & 40 & 72.7 & \multirow{2}{*}{$\begin{array}{c}1.19 \\
>0.05\end{array}$} \\
\hline & 20 & 37.0 & 15 & 27.3 & \\
\hline \multicolumn{6}{|l|}{ Education } \\
\hline \multirow{3}{*}{$\begin{array}{l}\text { Basic education } \\
\text { Secondary education } \\
\text { University education }\end{array}$} & 5 & 9.3 & 4 & 7.3 & \multirow{3}{*}{$\begin{array}{l}.552 \\
>0.05\end{array}$} \\
\hline & 30 & 55.6 & 28 & 50.9 & \\
\hline & 19 & 35.1 & 23 & 41.8 & \\
\hline \multicolumn{6}{|l|}{ Marital status } \\
\hline \multirow{3}{*}{$\begin{array}{l}\text { Married } \\
\text { Divorced } \\
\text { Widowed }\end{array}$} & 46 & 75.9 & 46 & 69.1 & \multirow{3}{*}{$\begin{array}{c}.524 \\
>0.05\end{array}$} \\
\hline & 3 & 5.6 & 2 & 36.6 & \\
\hline & 5 & 18.5 & 7 & 27.3 & \\
\hline \multicolumn{6}{|l|}{ Nature of work } \\
\hline \multirow{2}{*}{$\begin{array}{l}\text { Office and writing work } \\
\text { Require physical effort and movement }\end{array}$} & 52 & 96.3 & 49 & 89.1 & \multirow{2}{*}{$\begin{array}{c}2.08 \\
>0.05\end{array}$} \\
\hline & 2 & 3.7 & 6 & 10.9 & \\
\hline \multicolumn{6}{|l|}{ Monthly income } \\
\hline Enough & 12 & 22.2 & 19 & 34.5 & \multirow{2}{*}{$\begin{array}{c}2.03 \\
>0.05\end{array}$} \\
\hline Not enough & 42 & 77.8 & 36 & 65.5 & \\
\hline
\end{tabular}

Table (2): Mean score of anthropometric measurements of control and study groups $(n=109)$

\begin{tabular}{|c|c|c|c|c|}
\hline \multirow{2}{*}{ Parameters } & $\begin{array}{c}\text { Control group } \\
\mathbf{n = 5 4}\end{array}$ & $\begin{array}{c}\text { Study group } \\
\mathbf{n = 5 5}\end{array}$ & $\begin{array}{c}\text { Independent } \\
\text { t test }\end{array}$ & \multirow{2}{*}{ P value } \\
\cline { 2 - 3 } & Mean $\mathbf{\pm S D}$ & Mean \pm SD & & \\
\hline Weight in kg & $77.54 \pm 12.24$ & $80.95 \pm 11.24$ & -1.15 & $>0.05$ \\
Height in cm & $161.70 \pm 3.76$ & $160.33 \pm 4.63$ & .465 & $>0.05$ \\
BMI & $29.71 \pm 4.93$ & $31.12 \pm 4.30$ & -1.58 & $>0.05$ \\
\hline
\end{tabular}


Table (3): Distribution of the studied women (control and study groups) according to their knowledge regarding osteoporosis at pre and post intervention phases $(n=109)$.

\begin{tabular}{|c|c|c|c|c|c|c|c|c|c|c|}
\hline \multirow{3}{*}{ Knowledge items } & \multicolumn{5}{|c|}{ Before intervention } & \multicolumn{5}{|c|}{ After intervention } \\
\hline & \multicolumn{2}{|c|}{$\begin{array}{l}\text { Control } \\
\text { group }\end{array}$} & \multicolumn{2}{|c|}{$\begin{array}{l}\text { Study } \\
\text { group }\end{array}$} & \multirow{2}{*}{$\begin{array}{c}\text { X2 } \\
\text { p- } \\
\text { value }\end{array}$} & \multicolumn{2}{|c|}{$\begin{array}{l}\text { Control } \\
\text { group }\end{array}$} & \multicolumn{2}{|c|}{$\begin{array}{l}\text { Study } \\
\text { group }\end{array}$} & \multirow{2}{*}{$\begin{array}{c}\text { X2 } \\
\text { p-value }\end{array}$} \\
\hline & $\overline{\text { No }}$ & $\%$ & $\overline{\text { No }}$ & $\%$ & & $\overline{\text { No }}$ & $\%$ & $\overline{\text { No }}$ & $\%$ & \\
\hline \multicolumn{11}{|l|}{ Definition of osteoporosis } \\
\hline Complete correct answer & 7 & 13 & 10 & 18.2 & \multirow{3}{*}{$\begin{array}{l}0.981 \\
0.612\end{array}$} & 8 & 14.8 & 39 & 70.9 & \multirow{3}{*}{$\begin{array}{l}35.522 € \\
0.000^{* *}\end{array}$} \\
\hline Incomplet & 24 & 44.4 & 26 & 47.3 & & 26 & 48.1 & 11 & 20 & \\
\hline I don't know or wrong & 23 & 42.6 & 19 & 34.5 & & 20 & 37 & 5 & 9.1 & \\
\hline \multicolumn{11}{|c|}{ Factors that contribute to the occurrence of osteoporosis } \\
\hline Complete correct answer & 3 & 5.6 & 5 & \multirow{3}{*}{$\begin{array}{l}9.1 \\
34.5 \\
6.4 \\
\end{array}$} & \multirow{3}{*}{$\begin{array}{l}0.99 € \\
0.609\end{array}$} & \multirow{2}{*}{$\frac{5}{25}$} & 9.3 & 30 & 54.5 & \multirow{3}{*}{$\begin{array}{l}36.55 € \\
0.000 * *\end{array}$} \\
\hline \multirow{2}{*}{$\begin{array}{l}\text { Incomplete correct answer } \\
\text { I don't know }\end{array}$} & 16 & 29.6 & 19 & & & & 46.3 & 23 & 41.9 & \\
\hline & 35 & 64.8 & 31 & & & 24 & 44.4 & 2 & 3.6 & \\
\hline \multicolumn{11}{|l|}{ Symptoms of osteoporosis } \\
\hline Complete & 4 & 7.4 & 3 & 5.5 & \multirow{3}{*}{$\begin{array}{l}1.14 € \\
0.564\end{array}$} & 8 & 14.8 & 37 & 37 & \multirow{3}{*}{$\begin{array}{c}41.37 \\
0.000^{* *}\end{array}$} \\
\hline Incomplete correct answer & 23 & 42.6 & 29 & 52.7 & & 31 & 54.4 & 13 & 13 & \\
\hline I don't know & 27 & 50 & 23 & 41.8 & & 15 & 27.8 & 5 & 5 & \\
\hline \multicolumn{11}{|l|}{ Diagnosis of osteoporosis } \\
\hline Complete corre & 3 & 5.6 & 4 & 7.3 & \multirow{3}{*}{$\begin{array}{l}0.30 € \\
0.860\end{array}$} & 5 & 9.3 & 31 & 56.4 & \multirow{3}{*}{$\begin{array}{c}51.51 \\
0.000^{* *}\end{array}$} \\
\hline Incomplete correct answer & 18 & 33.3 & 20 & 36.4 & & 24 & 44.4 & 19 & 34.5 & \\
\hline I don't know & 33 & 61.1 & 31 & 56.3 & & 25 & 46.3 & 5 & 9.1 & \\
\hline tomoguroh & den: & & & & & & & & & \\
\hline Con & 0 & 0 & 2 & 3.6 & & 2 & 3.7 & 29 & 52.7 & \\
\hline Inc & 24 & 44.4 & 19 & 34.5 & 024 & 33 & 61.1 & 17 & 30.9 & 3L.2UE \\
\hline I don't know & 30 & 55.6 & 34 & 61.8 & & 19 & 35.2 & 9 & 16.4 & \\
\hline 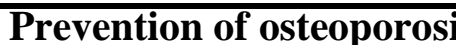 & & & & & & & & & & \\
\hline Com & 4 & 7.4 & 7 & 12.7 & & 3 & 5.6 & 49 & 89.1 & \\
\hline Incomplete correct answer & 18 & 33.3 & 21 & 38.2 & 0.481 & 21 & 38.9 & 6 & 10.9 & $0000 * *$ \\
\hline I don't know & 32 & 59.3 & 27 & 49.1 & & 30 & 55.6 & 0 & 0 & \\
\hline Methods of $p$ & ice & risk & 1a & & & & & & & \\
\hline Com & 6 & 11.1 & 7 & 12.7 & & 5 & 9.3 & 31 & 56.3 & \\
\hline Incor & 22 & 40.7 & 27 & 49.1 & 0 & 21 & 38.9 & 21 & 38.2 & 38. \\
\hline I don't know & 26 & 48.2 & 21 & 38.2 & & 28 & 51.8 & 3 & 5.5 & \\
\hline Treatment of & & & & & & & & & & \\
\hline Complete corr & 2 & 3.7 & 1 & 1.8 & & 0 & 0 & 18 & 32.7 & \\
\hline Incomplete correct answer & 15 & 27.8 & 21 & 38.2 & $\begin{array}{l}1.536 \\
0460\end{array}$ & 28 & 48.1 & 32 & 58.2 & $\begin{array}{c}34.64 t \\
0000 * *\end{array}$ \\
\hline I don't know & 37 & 68.5 & 33 & 60 & & 26 & 51.9 & 5 & 9.1 & \\
\hline
\end{tabular}

A Statistical significant $p \leq 0.05$

A Highly Statistical significant $\mathbf{p} \leq \mathbf{0 . 0 0 1}$

$*$ A statistical significant difference $(\mathrm{P} \leq \mathbf{0 . 0 0 5}) \quad * * A$ high statistical significant difference $(\mathrm{P}$ $\leq$ 0.001) $\quad €$ Fisher Exact Test

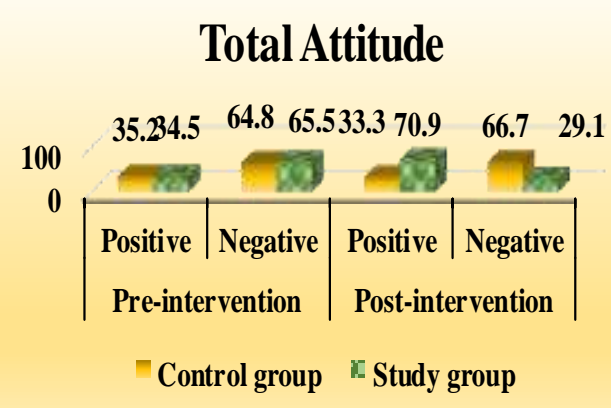

Figure (1): Distribution of the studied women (control and study groups) according to their total attitude score regarding prevention of osteoporosis at pre and post intervention phases 
Application of Precede-Proceed Model Based Education for Prevention of Osteoporosis Risks among Perimenopausal Women

Table (4): Distribution of the studied women (control and study groups) according to their healthy practices and activities regarding dietary habits at pre and post intervention phases $(\mathrm{n}=109)$.

\begin{tabular}{|c|c|c|c|c|c|c|c|c|c|c|}
\hline \multirow{3}{*}{ Dietary habits } & \multicolumn{5}{|c|}{ Before intervention } & \multicolumn{5}{|c|}{ After intervention } \\
\hline & & & & & $\begin{array}{l}\text { X2 } \\
\text { p- }\end{array}$ & & & & & $\begin{array}{l}\text { X2 } \\
\text { p- }\end{array}$ \\
\hline & No & $\%$ & No & $\%$ & & No & $\%$ & $\overline{\text { No }}$ & $\%$ & \\
\hline
\end{tabular}

Eating three regular meals a day that contain all the nutrients that are beneficial for the bones

\begin{tabular}{|c|c|c|c|c|c|c|c|c|c|c|}
\hline \multirow{3}{*}{$\begin{array}{c}\text { Always } \\
\text { Sometimes } \\
\text { Never }\end{array}$} & 7 & 13 & 8 & 14.5 & \multirow{3}{*}{$\begin{array}{l}0.452 \\
0.796\end{array}$} & 8 & 14.8 & 22 & 40 & \multirow{3}{*}{$\begin{array}{c}13.45 \\
0.000^{*} \\
*\end{array}$} \\
\hline & 29 & 53.7 & 26 & 47.3 & & 25 & 46.3 & 26 & 47.3 & \\
\hline & 18 & 33.3 & 21 & 38.2 & & 21 & 38.9 & 7 & 12.7 & \\
\hline
\end{tabular}

Eating eggs and dairy products

\begin{tabular}{|c|c|c|c|c|c|c|c|c|c|c|}
\hline \multirow{3}{*}{$\begin{array}{c}\text { Always } \\
\text { Sometimes } \\
\text { Never }\end{array}$} & 5 & 9.3 & 6 & 10.9 & \multirow{3}{*}{$\begin{array}{c}0.748 \\
€ \\
0.688\end{array}$} & 3 & 5.6 & 21 & 36.4 & \multirow{3}{*}{$\begin{array}{c}11.42 € \\
0.000^{*} \\
*\end{array}$} \\
\hline & 19 & 35.2 & 23 & 41.8 & & 27 & 50 & 23 & 43.6 & \\
\hline & 30 & 55.6 & 29 & 47.3 & & 24 & 44.4 & 11 & 20 & \\
\hline
\end{tabular}

Eating fresh fruits and vegetables that contain calcium and vitamins

\begin{tabular}{|c|c|c|c|c|c|c|c|c|c|c|}
\hline Alu & 5 & 9.3 & 3 & 5.5 & 0.855 & 10 & 18.5 & 18 & 32.7 & $10.67 €$ \\
\hline Sometimes & 24 & 44.4 & 25 & 45.4 & $€$ & 29 & 53.7 & 34 & 61.8 & $0.000 *$ \\
\hline Never & 25 & 46.3 & 27 & 49.1 & 0.754 & 15 & 27.8 & 3 & 5.5 & \\
\hline
\end{tabular}

Eating fish regularly

\begin{tabular}{|c|c|c|c|c|c|c|c|c|c|c|}
\hline \multirow{4}{*}{$\begin{array}{c}\text { Always } \\
\text { Sometimes } \\
\text { Never } \\
\end{array}$} & & & & & & & & & & \\
\hline & \multirow{3}{*}{$\begin{array}{c}5 \\
24 \\
25 \\
\end{array}$} & & & \multirow{2}{*}{$\begin{array}{l}5.5 \\
60\end{array}$} & \multirow{3}{*}{$\begin{array}{l}2.73 € \\
0.255\end{array}$} & 3 & 5.6 & 47.3 & 47.3 & \multirow{3}{*}{$\begin{array}{c}25.04 € \\
0.000 * \\
*\end{array}$} \\
\hline & & & & & & 30 & 55.6 & 20 & 36.3 & \\
\hline & & \begin{tabular}{|c|c|}
5 & 9.3 \\
24 & 44.4 \\
55 & 46.3 \\
\end{tabular} & $\begin{array}{l}33 \\
19 \\
\end{array}$ & $\begin{array}{c}60 \\
34.5 \\
\end{array}$ & & 21 & 38.9 & 9 & 16.4 & \\
\hline \multicolumn{11}{|l|}{ Eating legumes } \\
\hline \multirow{3}{*}{$\begin{array}{l}\text { Always } \\
\text { Sometimes } \\
\text { Never }\end{array}$} & 4 & 7.4 & 6 & 10.9 & \multirow{3}{*}{$\begin{array}{l}5.53 € \\
0.057\end{array}$} & 5 & 9.3 & 36 & 65.5 & \multirow{3}{*}{$\begin{array}{c}40.86 \\
0.000 * \\
*\end{array}$} \\
\hline & 18 & 33.3 & 29 & 52.7 & & 21 & 38.9 & 14 & 25.4 & \\
\hline & 32 & 59.3 & 20 & 36.4 & & 28 & 51.8 & 5 & 9.1 & \\
\hline
\end{tabular}

Using vegetable ghee / or oils instead of animal ghee in cooking food

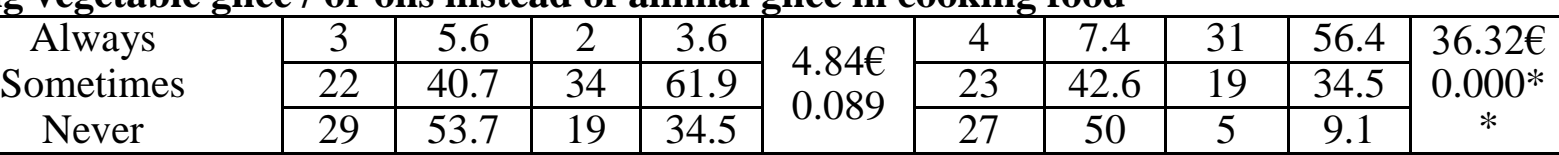

Reducing the intake of animal protein

Always

Sometimes

Never

\begin{tabular}{|c|c|c|c|}
\hline 2 & 3.7 & 5 & 9.1 \\
\hline 24 & 44.4 & 28 & 50.9 \\
\hline 28 & 51.9 & 22 & 40 \\
\hline
\end{tabular}

$2.30 €$

0.316

\begin{tabular}{|c|c|c|c|c|}
\hline 7 & 13 & 40 & 72.7 & $50.28 €$ \\
\cline { 1 - 4 } 21 & 38.9 & 11 & 20 & $0.000^{*}$ \\
\cline { 1 - 4 } 26 & 48.1 & 4 & 7.3 & $*$ \\
\hline
\end{tabular}

Avoid eating foods rich in fat

Always

Sometimes

Never

\begin{tabular}{|c|c|c|c|}
\hline 1 & 1.9 & 2 & 3.6 \\
\hline 24 & 44.4 & 28 & 50.9 \\
\hline 29 & 53.7 & 25 & 45.5 \\
\hline
\end{tabular}

0.928

$€$

0.629

\begin{tabular}{|c|c|c|c|c|}
\hline 2 & 3.7 & 24 & 43.6 & $25.37 €$ \\
\hline 28 & 51.9 & 21 & 38.2 & $0.000^{*}$ \\
\hline 24 & 44.4 & 10 & 18.2 & \\
\hline
\end{tabular}

Avoid eating pickles and salty foods

Always

Sometimes

Never

Avoid eating preserved foods

Always

Sometimes

Never

\begin{tabular}{|c|c|c|c|}
\hline 6 & 11.1 & 7 & 12.7 \\
\hline 36 & 66.7 & 43 & 78.2 \\
\hline 12 & 22.2 & 5 & 9.1 \\
\hline
\end{tabular}

$3.80 €$

0.150

\begin{tabular}{|c|c|c|c|c|}
\hline 9 & 16.7 & 47 & 85.5 & $54.53 €$ \\
\hline 25 & 46.3 & 8 & 14.5 & $0.000 *$ \\
\hline 20 & 37 & 0 & 0 & \\
\hline
\end{tabular}

3.571

0.168

\begin{tabular}{|c|c|c|c|c|}
\hline 8 & 14.8 & 36 & 65.4 & $29.04 €$ \\
\cline { 1 - 4 } & 70.4 & 16 & 29.1 & $0.000^{*}$ \\
\cline { 1 - 3 } & 14.8 & 3 & 5.5 & $*$ \\
\hline
\end{tabular}

Avoid taking fast food out of home

Always

\begin{tabular}{l|l}
10 & 18.2 \\
\hline
\end{tabular}

0.981

8

\begin{tabular}{l|l|}
14.8 & 39 \\
\hline
\end{tabular}

\begin{tabular}{|l|l|}
\hline 70.9 & $35.52 €$ \\
\hline
\end{tabular} 
$\underline{\text { Amany Ahmed, Mohamed Abd-Elsalm, Soad Abd-Elsalam and Hemmat Mostafa }}$

\begin{tabular}{|c|c|c|c|c|c|c|c|c|c|c|}
\hline \multirow{2}{*}{$\begin{array}{l}\text { Sometimes } \\
\text { Never }\end{array}$} & 24 & 44.4 & 26 & 47.3 & \multirow[t]{2}{*}{0.612} & 26 & 48.1 & 11 & 20 & \multirow{2}{*}{$\begin{array}{c}0.000^{*} \\
*\end{array}$} \\
\hline & 23 & 42.6 & 19 & 34.5 & & 20 & 37 & 5 & 9.1 & \\
\hline \multicolumn{11}{|c|}{ Avoid adding too much salt to food. } \\
\hline \multirow{3}{*}{$\begin{array}{c}\text { Always } \\
\text { Sometimes } \\
\text { Never }\end{array}$} & 6 & 11.1 & 9 & 16.4 & \multirow{3}{*}{$\begin{array}{l}0.994 \\
0.608\end{array}$} & 5 & 9.3 & 49 & 89.1 & \multirow{3}{*}{$\begin{array}{c}69.727 \\
€ \\
0.000^{*} \\
*\end{array}$} \\
\hline & 20 & 37 & 22 & 40 & & 24 & 44.4 & 4 & 7.3 & \\
\hline & 28 & 51.9 & 24 & 43.6 & & 25 & 46.3 & 2 & 3.6 & \\
\hline \multicolumn{11}{|c|}{ Avoid drinking soft drinks } \\
\hline \multirow{3}{*}{$\begin{array}{l}\text { Always } \\
\text { Sometimes } \\
\text { Never }\end{array}$} & 0 & 0 & 0 & \multirow{3}{*}{$\begin{array}{l}0 \\
3.6 \\
6.4\end{array}$} & \multirow{3}{*}{$\begin{array}{l}.238 € \\
0.626\end{array}$} & 0 & 0 & 22 & 40 & \multirow{3}{*}{$\begin{array}{c}46.87 € \\
0.000^{*} \\
*\end{array}$} \\
\hline & 16 & 29.6 & 13 & & & 18 & 33.3 & 23 & 41.8 & \\
\hline & 38 & 70.4 & 42 & & & 36 & 66.7 & 10 & 18.2 & \\
\hline \multicolumn{11}{|c|}{ Avoid drinking more than 2 cups of tea per day } \\
\hline \multirow{3}{*}{$\begin{array}{l}\text { Always } \\
\text { Sometimes } \\
\text { Never }\end{array}$} & 5 & 9.3 & 3 & \multirow{3}{*}{$\frac{5.5}{60}$} & \multirow{3}{*}{$\begin{array}{l}2.73 € \\
0.255\end{array}$} & 3 & 5.6 & 31 & 56.4 & \multirow{3}{*}{$\begin{array}{c}32.79 € \\
0.000^{*} \\
*\end{array}$} \\
\hline & 24 & 44.4 & 33 & & & 37 & 68.5 & 17 & 30.9 & \\
\hline & 25 & 46.3 & 19 & & & 14 & 25.9 & 7 & 12.7 & \\
\hline \multicolumn{11}{|c|}{ Avoid drinking more than 2 cups of coffee per day. } \\
\hline \multirow{3}{*}{$\begin{array}{l}\text { Always } \\
\text { Sometimes } \\
\text { Never }\end{array}$} & 0 & 0 & 2 & \multirow{3}{*}{$\begin{array}{l}3.6 \\
1.9 \\
4.5 \\
\end{array}$} & \multirow{3}{*}{$\begin{array}{l}4.84 € \\
0.089\end{array}$} & 0 & 0 & 30 & 54.6 & \multirow{3}{*}{$\begin{array}{c}58.19 € \\
0.000^{*} \\
*\end{array}$} \\
\hline & 15 & 27.8 & 23 & & & 16 & 29.6 & 19 & 34.5 & \\
\hline & 39 & 72.2 & 30 & & & 38 & 70.4 & 6 & 10.9 & \\
\hline \multicolumn{11}{|c|}{ Following a diet to maintain weight. } \\
\hline \multirow{3}{*}{$\begin{array}{l}\text { Always } \\
\text { Sometimes } \\
\text { Never }\end{array}$} & 0 & 0 & 2 & 3.6 & \multirow{3}{*}{$\begin{array}{l}3.135 \\
0.209\end{array}$} & 0 & 0 & 32 & 58.2 & \multirow{3}{*}{$\begin{array}{c}52.45 € \\
0.000^{*} \\
*\end{array}$} \\
\hline & 19 & 35.2 & 24 & 43.7 & & 24 & 44.4 & 19 & 34.5 & \\
\hline & 35 & 64.8 & 29 & 52.7 & & 30 & 55.6 & 4 & 7.3 & \\
\hline
\end{tabular}

*A statistical significant difference $(\mathrm{P} \leq \mathbf{0 . 0 0 5}) \quad * * A$ high statistical significant difference $(P$ $\leq$ 0.001) $\quad €$ Fisher Exact Test

Table (5): Correlation coefficient between total knowledge score, total attitude score and total healthy practices and activities of the studied women at pre and post-intervention phases $(\mathbf{n}=109)$.

\begin{tabular}{|c|c|c|c|c|c|c|c|c|}
\hline \multirow{4}{*}{ Variables } & \multicolumn{8}{|c|}{ Total Knowledge } \\
\hline & \multicolumn{4}{|c|}{ Control group } & \multicolumn{4}{|c|}{ Study group } \\
\hline & \multicolumn{2}{|c|}{$\begin{array}{c}\text { Before } \\
\text { intervention }\end{array}$} & \multicolumn{2}{|c|}{ After intervention } & \multicolumn{2}{|c|}{$\begin{array}{c}\text { Before } \\
\text { intervention }\end{array}$} & \multicolumn{2}{|c|}{ After intervention } \\
\hline & $\mathbf{r}$ & p-value & $\mathbf{r}$ & p-value & $\mathbf{r}$ & p-value & $\mathbf{r}$ & p-value \\
\hline Total attitude & $\begin{array}{c}0.83 \\
8 \\
\end{array}$ & $\begin{array}{c}\leq \\
0.001 * *\end{array}$ & $\begin{array}{c}0.67 \\
4 \\
\end{array}$ & $\begin{array}{c}\leq \\
0.001 * *\end{array}$ & $\begin{array}{c}0.73 \\
3 \\
\end{array}$ & $\begin{array}{c}\leq \\
0.001 * *\end{array}$ & $\begin{array}{c}0.75 \\
9 \\
\end{array}$ & $\begin{array}{c}\leq \\
0.001 * *\end{array}$ \\
\hline $\begin{array}{l}\text { Total healthy } \\
\text { practices and } \\
\text { activities }\end{array}$ & $\begin{array}{c}0.73 \\
9\end{array}$ & $\begin{array}{c}\leq \\
0.001 * *\end{array}$ & $\begin{array}{c}0.74 \\
5\end{array}$ & $\begin{array}{c}\leq \\
0.001 * *\end{array}$ & $\begin{array}{c}0.51 \\
5\end{array}$ & $\begin{array}{c}\leq \\
0.001 * *\end{array}$ & $\begin{array}{c}0.54 \\
9\end{array}$ & $\begin{array}{c}\leq \\
0.001 * *\end{array}$ \\
\hline
\end{tabular}

A statistical significant difference $(\mathrm{P} \leq 0.005) \quad * *$ A high statistical significant difference $(\mathrm{P} \leq$ 0.001) $\quad €$ Fisher Exact Test 


\section{Discussion}

Peri-menopause represents a delicate transition period of a woman's life during which physiological, affective, psychological, and social changes mark progression from a woman's fertile life to climaterium, with wide sexual hormones fluctuations until the onset of hypergonadotropic amenorrhea. During this stage, because of the decreases or fluctuations of sex hormones caused by recession of ovarian function, women undergo a series of psychological and physical symptoms, clinically called perimenopausal syndrome Huang et al, (2020).

Socio-demographic characteristics of studied women such as age, residence, educational level, occupation and income mainly affect women's knowledge, attitude, practices, enabling factors and reinforcing factors regarding prevention of osteoporosis risks. So, these factors should be determined for the studied women. The finding of the current study revealed that more than twofifths and more than one-third of both study and control groups respectively in age group ( $46<50$ years) with a mean age of $47.67 \pm 2.34$ years and $48.41 \pm 3.54$ years respectively.

Concerning the body mass index, the results of current study represented the anthropometric measurements of both study and control group, it showed that, the mean weight of both study and control groups were $80.95 \pm 11.24 \mathrm{~kg}$ and $77.54 \pm 12.24 \quad \mathrm{~kg}$ respectively, the mean height of both study and control groups were $160.33 \pm 4.63 \mathrm{~cm}$ and $161.70 \pm 3.76 \mathrm{~cm}$ respectively. In addition, the mean body mass index in both study and control groups were $31.12 \pm 4.30 \mathrm{~kg} / \mathrm{m} 2$ and $29.71 \pm 4.93 \mathrm{~kg} / \mathrm{m} 2$ respectively, this mean no significance difference in anthropometric data of both group ( $p>0.05)$ reflect homogenous.
Additionally the result of the current study was consistent with Mallhi et al, (2019) who conducted "Knowledge, Attitude and Practice (KAP) Survey of Osteoporosis among Students of a Tertiary Institution in Malaysia", found that a minority of participants reported that health care professionals were their primary source of information. This may be due to lack of time on the part of health care professional, ignorance of the seriousness of osteoporosis as a disease or even low level of knowledge among healthcare professionals. Studies that evaluated knowledge of osteoporosis among healthcare professionals surprisingly reported a low level of knowledge especially among nurses who are more often in contact with patients and their families Chen et al., (2017).

Additionally, EITohami, et al, (2015) in a study entitled "Study of Knowledge, Attitude and Practice of Osteoporosis among Adult Women in Majmaah City, Saudi Arabia", revealed that Media was the main source of information about osteoporosis.

In the same context, Hajizadeh et al, (2019) who studied "Development and validation of a theory of planned behaviorbased weight control behavior questionnaire among postmenopausal women with osteoporosis", concluded that postmenopausal women, deprived of the protective effects of endogenous estrogen are predisposed to increases in weight. For instance, women have exhibited a sharp increase in obesity between the ages of 45 and 54 years, as a result of declines in endogenous estrogen, together with physical. Overweight or high body mass index (BMI) are also reported as risk factors for vertebral fracture among postmenopausal osteoporotic women.

Owing to knowledge of the studied women regarding prevention of osteoporosis risks at menopause, the current study findings 
clarified that there was no statistically significant difference between control and study groups regarding all items of knowledge about menopause, osteoporosis, hormonal replacement therapy, dietary habits, practicing sports, importance of sun exposure and periodic examination at menopause before intervention $(\mathrm{P}>0.05)$. However, there was a highly statistical significant improvement were observed in the study group compared with the control group after intervention $(p<0.001)$. Where, it was displayed that, one-fifth of study group and less than one-quarter of control group had good knowledge before intervention. Meanwhile, after intervention, more than three-quarters of study group had good knowledge compared with more than one-quarter $(27.7 \%)$ of the control group.

This result was consistent with Tabasi et al, (2018) who conducted "Application of the Precede-Proceed Model in Promoting Physical Activity for Prevention of Osteoporosis among Women" found that after educational interventions, the mean scores of predisposing factors including knowledge are significantly increased only in the intervention group $(p<0.001)$. In this regard, the present study was consistent with studies conducted by Estebsari et al,(2019), and Polcyn et al, (2019) who studied " Planning and evaluation of an educational program based on Precede model to improve physical activity in female students", "Eliminating iodine deficiency disorders in Nepal through Precede-Proceed" and "Utility of the Precede model in differentiating users and nonusers of smokeless tobacco" respictively. These findings reflect the impact of the implementation of PPM model-based interventions on raising the level of knowledge among people, especially regarding the fact that knowledge is of utmost importance in making the right attitude and behavior.
Moreover, Jeihooni et al, (2019) in a study entitled "The effect of education based on based health belief model on osteoporosis and bone mineral density among women", reported that the experimental group showed a significant increase in knowledge, perceived susceptibility, perceived severity, perceived benefits, perceived barriers, self-efficacy, internal cues to action, nutrition, and walking performance immediately and one year after the intervention. The result obtained from these studies can be concluded that providing educational programs in this regard for family members, physicians and other health personnel and offering training programs in radio and television broadcasting is essential.

Additionally this result went in the same line with Pourhaji et al, (2020) in the study entitled "Effects of educational program based on Precede-Proceed model in promoting low back pain behaviors (EPPLBP) in health care workers Shahid Beheshti University of medical sciences: randomized trial", reported that there was a significant interaction between the factors "group" and "test time" $(\mathrm{p}<0.05, \mathrm{p}$ $<0.001$ ) of knowledge of the intervention group., although, no significant alternate became located in the mean score of above structures of the control group.

Also, the result of the current study was supported by El-Said et al, (2021) who carried out study on "Application of an Educational Program on Lifestyle of Perimenopausal Women Utilizing PRECEDE - PROCEED Model", revealed that there was no statistically significant difference between control and study groups regarding knowledge before PPM application. However, there was a highly statistically significant difference was observed in knowledge of the study group compared with the control group after three and six months of PPM application.

The findings confirmed the effectiveness of the PRECEDE-PROCEED 
model-based educational program on preventing osteoporosis by enhancing scores of model constructs. However, these results should be repeated in further studies to be able to apply this program in health system.

Increasingly, the result of the current study was supported by Sullivan, et al, (2017) in a study entitled "Psycho-educational intervention for achieving and maintaining adequate dietary calcium intake in premenopausal Chinese women", demonstrated that on controlling for the effect of age, no significant difference between the experimental and control group was found at the pre-test for knowledge. After the intervention, the experimental group scored significantly higher than the controls for knowledge on the immediate, 3-month and 6month post-tests.

It was reported by John Cecily, (2020) that majority of the women $94.5 \%$ had inadequate knowledge on preventive measures of osteoporosis. This indicates the ultimate importance of educational intervention to improve knowledge and thus prevent development of osteoporosis in later life. This result was in agreement with Hurst and Wham, (2007) who illustrated that there was lack of knowledge of the studied sample regarding prevention of osteoporosis risks.

Concerning attitude of the studied women regarding prevention of osteoporosis risks at menopause, there was no statistically significant difference between control and study groups regarding all items of attitude towards prevention of osteoporosis before intervention $(\mathrm{P}>0.05)$. However, there was a highly statistically significant improvement was observed in the study group compared with the control group after intervention $(\mathrm{p}<$ $0.001)$. Where, it was illustrated that, less than one-third of both study and control groups respectively had positive attitude before intervention. Meanwhile, after intervention, less than three-quarters of study group had positive attitude compared with less than onethird of the control group.

This result agreed with Estebsari al, (2019), indicated that after educational interventions, the mean scores of attitude significantly improved only in the intervention group ( $\mathrm{p}<0.001)$.

This result went in the same line with Pourhaji et al, (2020) reported that there was a significant interaction between the factors "group" and "test time" ( $p<0.05, \mathrm{p}<0.001)$ of attitude of the intervention group. Although, no significant alternate became located in the mean score of above structures of the control group. Increasingly, the result of the current study was supported by Sullivan, et al, (2017), demonstrated that on controlling for the effect of age, no significant difference between the experimental and control group was found at the pre-test for attitude. After the intervention, the experimental group scored significantly higher than the controls for attitude on the immediate, 3-month and 6-month post-tests. This result was in agreement with Hurst and Wham, (2007) who indicated that there was low score of attitude of the studied sample regarding prevention of osteoporosis.

Also, the result of the current study was supported by El-Said et al, (2021) who revealed that there was no statistically significant difference between control and study groups regarding attitude before PPM application. However, there was a highly statistically significant difference was observed in attitude of the study group compared with the control group after three and six months of PPM application.

In the same context Hurst and Wham, (2007) in a study entitled "Attitudes and knowledge about osteoporosis risk prevention: a survey of New Zealand women", these women demonstrated average levels of knowledge about osteoporosis risk factors. 
They had low feelings and attitude of susceptibility towards development of osteoporosis, but most considered it to be a serious disease.

Regarding healthy practices and activities of the studied women regarding prevention of osteoporosis risks at menopause, the current study findings clarified that there was no statistically significant difference between control and study groups regarding all items of healthy practices and activities regarding dietary habits, practicing exercise, exposure to the sun, smoking and periodic check-up, taking medication and reducing the risk of falling before intervention $(\mathrm{P}>0.05)$.However, there was a highly statistical significant improvement was observed in the study group compared with the control group after intervention ( $p<0.001)$. Where, it was displayed that, more than onequarter and less than one-third of both study and control groups respectively had satisfactory level of healthy practices and activities before intervention. Meanwhile, after intervention, less than two-thirds of study group had satisfactory level compared with more than one-quarter of the control group.

In the same context, Tabasi et al, (2018), found that after the intervention, there was a significant difference in the mean scores of practices and physical activity in the intervention group, which is probably because of the effects of the intervention. This is consistent with the studies of Lesan et al, (2018), and Shakouri et al, (2019) and Turner et al, (2017) who carried out studies on "Application of Precede in reducing Tehranian firemen anxiety", "Planning and evaluation of an educational program based on Precede model to improve physical activity in female students", " Effect of health education program base on Precede model in controlling iron-deficiency anemia among high school girl students in Talesh" and "Design and implementation of an osteoporosis prevention program using the health belief model" respectively.

Also, the result of the current study was supported by El-Said et al, (2021) who revealed that there was no statistically significant difference between control and study groups regarding practices and health promoting lifestyle before PPM application. However, there was a highly statistically significant difference was observed in practices and health promoting lifestyle of the study group compared with the control group after three and six months of PPM application.

This result went in the same line with Pourhaji et al (2020), reported that there was a significant interaction between the factors "group" and "test time" ( $p<0.05, \mathrm{p}<0.001)$ of quality of life and osteoporosis preventive behaviors of the intervention group, although, no significant alternate became located in the mean score of above structures of the control group.

Increasingly, the result of the current study was supported by Sullivan, et al, (2017), demonstrated that on controlling for the effect of age, no significant difference between the experimental and control group was found at the pre-test for behavioral change. After the intervention, the experimental group scored significantly higher than the controls for stage of behavioral change on the immediate, 3month and 6-month post-tests.

This result was in agreement with Hurst and Wham, (2007), who illustrated that there was lack of awareness about osteoporosis. Practice score was the most worrying condition compared with the two other domains (i.e., knowledge and attitude). Sharifi et al, (2017) who conducted "The Effects of an Educational Intervention Based on the Health Belief Model and SelfRegulation on Women's Eating Behaviors during Menopausal Transition", indicated that 
the educational intervention improved women's knowledge, attitudes, and healthy practices.

Within the PRECEDE-PROCEED Model, enabling and reinforcing factors are defined as factors that make it possible (or easier) for individuals or populations to change their behavior or their environment. Enabling factors include resources, conditions of living, societal supports, and skills that facilitate a behavior's occurrence. So, it is important to ascertaining which factors have the ability to cause each of these behaviors to occur or to inhibit their occurrence. These factors are themselves grouped into three types: predisposing, reinforcing, and enabling factors Simeon et al, (2020).

Increasingly, the result of the current study was consistent with Khan et al, (2014) who mentioned that there was lack of awareness about osteoporosis and unsatisfactory level of performance about osteoporosis prevention measures among study participant. The results of present study revealed an urgent need of a targeted educational and awareness program for women belonging to different age groups. Educational programs based on the parameters of the PRECEDE-PROCEED Model should be designed and implemented on a large scale

Concerning the correlation between total knowledge, total attitude and total healthy practices and activities, the results of the present study showed that there was that there was a highly statistically significant positive correlation between total knowledge score and total attitude of both study and control groups before and after intervention $(\mathrm{P}<0.001)$. Moreover, there was a highly statistically significant positive correlation between total knowledge score and total healthy practices and activities of both study and control groups before and after intervention $(\mathrm{P}<0.001)$.
The result of the current study was supported by El-Said et al. (2021) who revealed that there was no statistically significant difference between control and study groups regarding practices and health promoting lifestyle before PPM application. However, there was a highly statistically significant positive correlation between total health-promoting lifestyle score and educational and ecological assessment phase structures of PRECEDE - PROCEED model (predisposing, reinforcing, and enabling factors) of both groups before, after three and six months of program application $(\mathrm{P}<0.00)$. These results illustrated that the improvement of knowledge could lead to improvement of practice and affect attitude positively and vice versa

Increasingly, the result of the current study was consistent with Khan et al, (2014) who mentioned that a correlation between different domains of questionnaire was also assessed. A positive correlation was observed between knowledge and attitude, a positive correlation was observed between knowledge and practices. In the same context Hurst and Wham, (2017) demonstrated that there was a significant correlation was found between knowledge and health motivation, and belief in the benefits of calcium and exercise for the prevention of osteoporosis was highly predictive of health motivation scores. Also, Alshammari, (2014) reported that there was a significant correlation was found between their knowledge scores and their attitude level scores.

\section{Conclusion}

Application of PRECEDE-PROCEED model based education was effective in prevention of perimenopausal women's predisposing factors of osteoporosis, healthy practices and activities, reinforcing factors, enabling factors which supported the present study hypothesis. 


\section{Recommendations}

* Educational programs intended for women in the climacteric period must be extended about menopause, symptoms, long term consequences, and effective ways of dealing with it and such education should be supplemented with an educational manual or similar materials.

* Integration of PECEDE-PROCEED Model Based health education into the curriculum of nursing colleges.

* Health education programs concerning bone health for perimenopausal women should be strengthened to raise awareness regarding the association between menopause and bone mineral density.

*Emphasize the importance of routine measurement of bone mineral density and serum calcium level every year for all pre and post-menopausal females is needed for early detection and prevention of osteoporosis.

\section{Further researches:}

* It is crucial to conduct an extensive future studies with larger sample probability sizes of perimenopausal women from different regions of Egypt to enhance the accuracy of the findings and be able to apply PRECEDE-PROCEED model based intervention program in health system to increase the level of awareness towards osteoporosis.

\section{References}

Abd El Rahman , A,Abd El Hamed ,H.s and Ramadan,S.A(2019).Osteoporosis education programs: change knowledge and behavior among 20-60 years old females. Bulletin of High Institute of public Health. 33 (3):601-616

Alshammari, K.F. (2014). Women Knowledge, Attitude and Practices About Osteoporosis Prevention"Riyadh Saudi
Arabia", World Journal of Medical Sciences 11 (3): 422-431

Azar, F. E., Solhi, M., Darabi, F., Rohban, A., Abolfathi, M., \& Nejhaddadgar, N., (2018). Effect of educational intervention based on PRECEDE-PROCEED model combined with self-management theory on self-care behaviors in type diabetic patients. Diabetes \& Metabolic Syndrome: Clinical Research \& Reviews, 12 (6): 1075- 1078.

Chen.J, Wang S.T, Cheng S.P and Hua, L. (2017). Huang. Knowledge about osteoporosis and its related factors among public health nurses in Taiwan. Osteoporosis Int; 16(2): 2142-2148

El-Said,C Morse, $R$ \& Aeed ,A (2021). Osteoprosis :Egyptian womens life style and their quality of life 8 th European Congress on menopause63(1):S1-S136

EITohami, K, Sami ,W, AL Eidan A, AL Mubarak M, Alotaibi F. (2015). Study of Knowledge, Attitude and Practice of Osteoporosis among Adult Women in Majmaah City, Saudi Arabia, International Journal of Health and Rehabilitation Sciences, 4(3):185193

Estebsari F, Shojaeizadeh D, Mostafaei D and Farahbakhsh (2019). Planning and evaluation of an educational program based on Precede model to improve physical activity in female students. Hayat;16(1):48-54.

Falkingham, J., Evandrou, M., Qin, M., \& Vlachantoni, A., (2020). Chinese women's health and wellbeing in middle life: Unpacking the influence of menopause, lifestyle activities and social participation, Maturitas, 143, 145 150 .

Ghaderi, E., Ghazanfarpour, M., Kaviani, M., (2010). Evaluation of menopausal women's attitudes towards menopause in Shiraz. Pak, J Med Sci., 26 (3): 698-703. 
Hajizadeh, H., Nadrian, H., Farin, N., Jafarabadi, M. A., Hezaveh, S. J. G., Kolahi, S. and Brennan-Olsen, S. (2019). Development and validation of a theory of planned behavior-based weight control behavior questionnaire among postmenopausal women with osteoporosis. Health care for women international, 40(10), 1101-1116.

Hazavehei, S.M.M, Oruogi, M.A, Charkazi, A and Hassanzadeh A,. (2019). The effect of health education intervention based on Precede framework on modification of vegetable oils consumption habits in families under the cover of health centers in Mani Shahr of Khomein. J Arak Univ Med Sci;13(4):133-42.

Huang Z, Shi J, Liu W, Wei S and Zhang Z. (2020). The influence of educational level in peri-menopause syndrome and quality of life among Chinese women. Gynecological Endocrinology, 1-6

Hurst, P.R and Wham, C.A., (2017). Attitudes and knowledge about osteoporosis risk prevention: a survey of New Zealand women, Public Health Nutrition: 10(7), 747753

Jakimiuk, H., \& Jackson, C., (2020). Behavioral science theory and principles for practice in health education. Health Educ Res. 12 (1):143-150.

Jalili, Z., Didehvar, M., Zareban, I., Bakhshani ,N.M., \& Shahrakipour, M., (2021). The effect of stress management education based on Precede model on occupational stress of nurses in hospital of Iranshahr, Iran. Strides Dev Med Educ; 12(3):472-84.

Jeihooni, AK, Kashfi SM, Khiyali Z, Jamshidi $H$ and Kashfi SH (2019). The effect of education based on based health belief model on osteoporosis and bone mineral density among women, Journal of Research \& Health,
Social Development \& Health Promotion Research Center, 9(1): 11- 20

John,H.S., (2020). Early Detection and Prevention of Osteoporosis among Pre- and Postmenopausal Women in Saudi Arabia, Clinical Nursing Research, 29(1) 48- 55

Khan ,Y.H, Sarriff ,A, Khan, A.H and Mallhi ,T.H. (2014). Knowledge, Attitude and Practice (KAP) Survey of Osteoporosis among Students of a Tertiary Institution in Malaysia, Tropical Journal of Pharmaceutical Research January 2014; 13 (1): 155-162

Kuar, G., Kueh, Y. C., Abdullah, N., \& Tai, E. L. M., (2019). Psychometric properties of the health-promoting lifestyle profile II: Cross-cultural validation of the Malay language version, BMC public health, 19(1): 1-10.

Lesan Sh, Ghofranipour F, Birashk B and Faghihzadeh S. (2018). Application of Precede in reducing Tehranian firemen anxiety. Iran J Psychiatry Clin Psychol. 2003;9(2):77-83.

Mallhi, H., Hsieh, C., Novielli, K.D., Diamond, J.J., Cheruva, D., (2019). Health beliefs and attitudes toward the prevention of osteoporosis in older women. Menopause 8:372-376

Mckinney ,K.L., (2020). A meta-analysis of the effect of cigarette smoking on bone mineral density calcified tissue international,68:259-270.

Orabi, E.E., (2017). Effect of Health Education Intervention on Knowledge, and attitude regarding Menopausal Period among Premenopausal Female Employees. The Egyptian Journal of Community Medicine Vol. 35 No. 3: 71-84

Polcyn MM, Price JH, Jurs SG and Roberts SM. (2019). Utility of the Precede model in 
differentiating users and nonusers of smokeless tobacco. J Sch Health; 61(4):166-71.

Pourhaji F, Delshad MH, Tavafian SS, Niknami S and Pourhaji F (2020). Effects of educational program based on Precede-Proceed model in promoting low back pain behaviors (EPPLBP) in health care workers Shahid Beheshti University of medical sciences: randomized trial, Heliyon 6 (2020) e05236, available at: www.cell.com/heliyon

Recker,K., (2019).Lifestyle and osteoporosis fracture in middle aged women in alexandaria.journal of the medical research institute, 19(2):83-104.

Shakouri, S., Sharifi Rad, G.R., Golshiri ,P., Hassanzadeh ,A., \& Shafe Shakouri, M., (2019). Effect of health education program base on Precede model in controlling irondeficiency anemia among high school girl students in Talesh. J Arak Univ Med Sci; 12(3):47-56.

\section{Sharifi, F., Mitra,T., Moodi,I., Gholamreza Sharifzade, \& Hakimeh, Malaki, Moghadam., (2017). The Effects of an Educational Intervention Based on the Health Belief Model and Self-Regulation on Women's Eating Behaviors During}

Menopausal Transition, Mod Care J; 14(2):e65305.

Simeon, R., Dewidar, O., Trawin, J., Duench, S., Manson, H., Pardo, J.P., Petkovic, J., Roberts, J.H., Tugwell, P., \& Yoganathan, M., (2020). Behavior change techniques included in reports of social media interventions for promoting health behaviors in adults: S5-S12

Sullivan, P.L, Wong, F.Y.Y and Chan, S.SC. (2017). Psycho-educational intervention for achieving and maintaining adequate dietary calcium intake in pre-menopausal Chinese women, Hong Kong Medical Journal, 2007, 13(2): S44-S47.

Tabasi Nejad, N., Mohseni, M., Khanjani, N., \& Ahmadi Tabatabaei, S. V. (2018). Application of the PRECEDE-PROCEED model in promoting physical activity for prevention of osteoporosis among women. Health Education and Health Promotion, 6(3), 103-108.

Turner LW, Hunt SB, Di Brezzo $R$ and Jones C. (2017). Design and implementation of an osteoporosis prevention program using the health belief model. Am J Health Stud.; 19(2):115-21. 
تطبيق نموذج السلوك الصحى المعتمد على التعليم لمنع مخاطر هثاثة العظام لاى السيدات حول سن الاياس

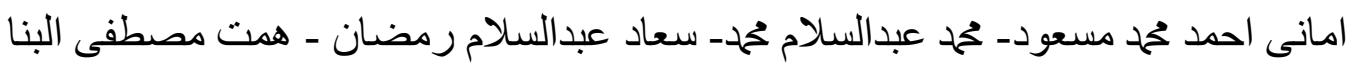

تعتبر صحة المر أة في مختلف الفئات العمرية من القضايا المهمة للوصول إلى الكفاءة في أداء جميع

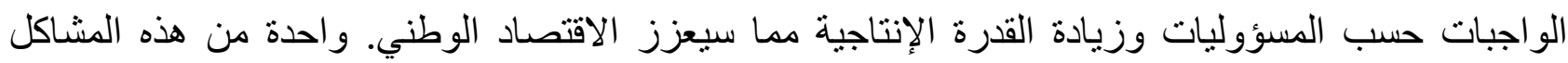

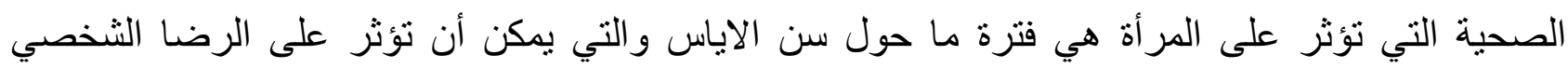

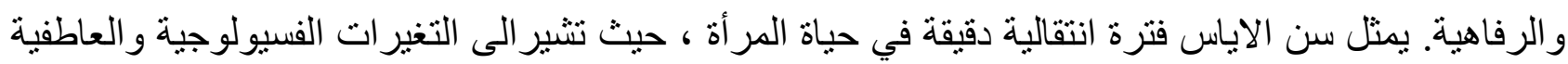

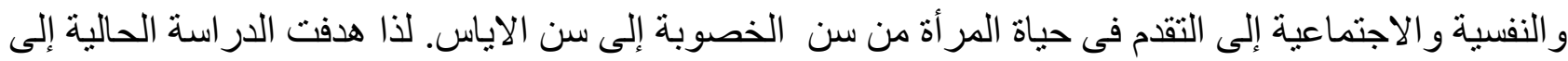

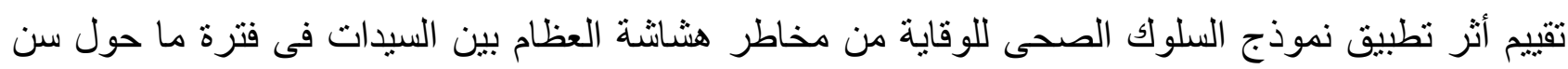
اليأس. وقد أجريت الدراسة في مستشفى جامعة بنها (موظفات إداريات) استنادًا إلى نتائج الدراسة الحالية ، تم استنتاج أن فرضية البحث تم دعمها وأن نموذج السلوك الصحى لة تأثير كبير على تحسين العوامل المؤهبة

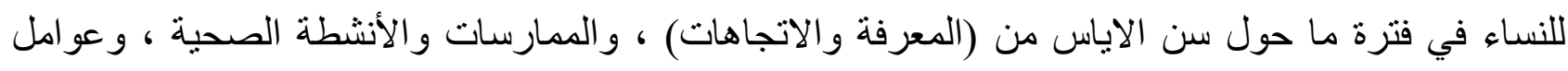

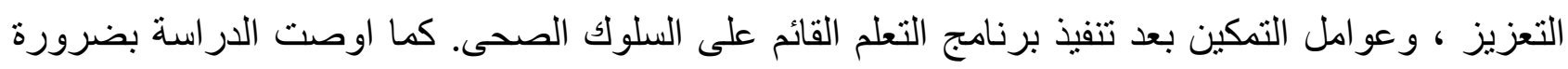

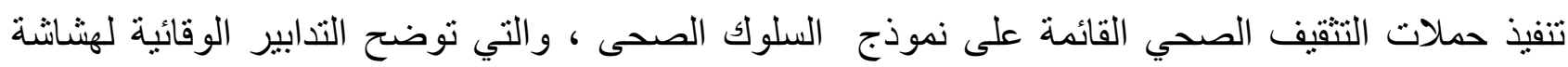
العظام لدى السيدات حول فترة سن الاياس. 